\title{
Influence of the Axial Turbine Design Parameters on the
} Stator-Rotor Axial Clearance Losses

\author{
Aki Grönman ${ }^{1}$, Teemu Turunen-Saaresti ${ }^{2}$, Pekka Röyttä ${ }^{3}$ and Ahti Jaatinen-Värri \\ ${ }^{1}$ Corresponding author, Post-Doctoral Researcher, aki.gronman@ lut.fi, Institute of Energy, Lappeenranta \\ University of Technology, P.O. Box 20, FI-53851 Lappeenranta, Finland, Telephone +358 407767999 \\ ${ }^{2}$ Associate Professor, Institute of Energy, Lappeenranta University of Technology P.O. Box 20, FI-53851 \\ Lappeenranta, Finland \\ ${ }^{3}$ Researcher, Computational Engineering and Design, Fraunhofer Chalmers Centre, Chalmers Science Park, \\ SE-412 88 Gothenburg, Sweden
}

\begin{abstract}
The drive towards lower emissions in aerospace engines promotes more efficient and physically smaller engines. One way to decrease the size of the axial turbine is to minimize the distance between successive stator and rotor rows. This can usually have either a positive or negative influence on the turbine performance. The reasons for this behaviour are not currently fully understood. In this study, a novel approach is taken to find new insights into this design question by analysing the influence of different design parameters on the turbine efficiency behaviour. Several different turbines are analysed using the literature. For the first time, the performed analysis reveals the design parameters which correlate with the different efficiency curve shapes. The correlating parameters are the stator-rotor axial clearance, stator pitch to axial chord ratio, turning velocity Mach number, and rotor aspect ratio. The mechanisms behind the found correlations are further analysed to connect the physical phenomena with the design parameters.
\end{abstract}


Keywords: Axial turbine, axial clearance, axial gap, turbine efficiency

\section{I.Introduction}

Axial turbines are widely used in gas turbines, condensing power plants, and aero-engines. Quite often the space and the weight of a turbine are limited, which generates one constraint for the designer. These constraints are especially important in aero-engines and other transportation systems. One way to decrease the physical size of a turbine is to decrease the axial clearance between the stator and the rotor. This can, however, affect both the performance ${ }^{1,2}$ and the blade excitation. ${ }^{3}$ Additionally, it can also affect the axial force of the rotor wheel. ${ }^{4}$

Axial clearance can be considered as a significant location of losses in a turbine stage. The importance of the losses generated in the space between stator and rotor has been shown by Mokulys et al. ${ }^{5}$ Their results indicated that the axial clearance losses are the second highest source of losses after the stator profile loss in the studied two-dimensional turbine designs. It is, however, not clear how large part these losses play in the three-dimensional turbine stage loss breakdown. Overall, it can be estimated that the axial gap changes can lead to turbine stage efficiency changes of few percentage points.

The effect which the axial clearance has on the turbine performance is not always similar, and the loss mechanisms can differ between turbines. In some studies, the efficiency decreases as the clearance increases. ${ }^{1,6,7}$ However, some studies suggest a larger clearance to be more beneficial in terms of efficiency. ${ }^{8-}$ 10

The influence of the end walls and stator-rotor interaction in the end wall regions on the loss generation has been found to be important in many of the studied turbines. Restemeier et al. ${ }^{11}$ studied a 1.5 -stage low aspect ratio axial turbine both numerically and experimentally and found that the performance improves with 
a decreasing axial clearance. They concluded that the efficiency increase is mainly attributed to the end walls. They also concluded that their results imply shorter clearance to be more beneficial for turbines having a low aspect ratio. Additionally, Funazaki et al. ${ }^{2}$ studied the effect of changing axial clearance, and they noticed almost linear degradation of efficiency as the clearance was increased. They concluded that the increase of the axial clearance resulted in hub and tip boundary layer growth before the rotor. Yamada et al. ${ }^{12}$ found that at the design conditions the less beneficial wake interaction near the tip reduced the positive interaction near the hub and caused the non-linear efficiency behaviour when the axial clearance was increased. Gaetani et al. ${ }^{13}$ studied two axial clearances and found that in the low stator-rotor interaction tip region, the increased axial clearance increased losses, and this behaviour was opposite at the hub. Overall, the efficiency was better with the smaller axial clearance. Yamada et al. ${ }^{14}$ found an increase in the efficiency at the tip region with the increasing axial clearance in a steam turbine stage. Opposite behaviour was found from hub to midspan with overall lower efficiency in the large axial clearance case. The reason for lower efficiency near the end wall was attributed to the passage vortex, indicating fewer vortexes with the small axial clearance. Additionally, a study of Chang and Tavoularis ${ }^{15}$ predicted that the interaction between the stator wake and end wall boundary layer caused an increase in the entropy before the rotor.

In the study of Gaetani et al., ${ }^{16}$ large efficiency differences were observed at the tip region when the axial clearance was increased due to the development of casing end wall boundary layer. The wake induced interaction was found to decrease the efficiency with larger clearances and the vortex induced interaction with small clearances. They also suggested that at least with subsonic turbine stages the axial clearance should be optimized by controlling the superposition of the stator potential field and wake to minimize the rotor incidence fluctuation. 
The importance of wake mixing losses is mentioned in several studies. A study of Park et al. ${ }^{7}$ predicted decreasing efficiency when the axial clearance was increased. The decrease was attributed to be due to wake mixing loss increase with increasing axial clearance. In the second part of Venable et al., ${ }^{8}$ Busby et al. ${ }^{19}$ found that the increased stator losses with decreasing axial clearance were due to stronger stator-rotor interaction and wake mixing loss reduction. On the other hand, the increase in the stator wake and rotor blade interaction was mainly causing the increased blade relative total pressure losses.

Baagherzadeh Hushmandi and Fransson ${ }^{4}$ studied two-stage low reaction turbine with different axial clearances at partial admission and found a decrease in the first stage efficiency when the clearance was increased. They concluded that the reduced interaction between the main flow and the leakage flow inside the disk cavity caused the better stage efficiency with the smallest axial clearance. With the increased axial clearance the stage efficiency decreased due to the extra losses associated with the mixing of the main flow and disk cavity leakage flow.

In supersonic or transonic flows, the trailing edge shocks generate additional losses and complexity to the flow fields. Grönman and Turunen-Saaresti ${ }^{6}$ studied numerically the effect of axial clearance on the performance of a supersonic axial turbine. They found that the efficiency increases while the clearance decreases. It was concluded that this was caused by the increased total pressure losses in the axial clearance which was most likely due to the increased mixing between the main flow and stator wakes and shock waves. It also supported the earlier conclusion made by Jeong et al. ${ }^{20}$ for a supersonic impulse turbine in their numerical and experimental study.

The intensity of stator trailing edge shock seems to decrease with the increasing axial clearance as the study of Denos et al. ${ }^{21}$ suggests. However, the importance of trailing edge shock to the overall losses is not necessarily high in all turbines as a study of Chang and Tavoularis ${ }^{15}$ predicted that the entropy generation of 
the trailing edge shock is small compared to the wake. Griffin and Dorney ${ }^{22}$ studied supersonic axial turbine numerically and found that the power of the turbine was higher with the smaller clearance. The pressure unsteadiness at the rotor leading edge was found to decrease while increasing the axial clearance. The interaction between successive nozzle jets due to longer expansion was concluded to be a source of additional losses with the larger axial clearance.

The rotor flow unsteadiness is also affected by the axial clearance variation. Denos et al. ${ }^{21}$ found that the rotor leading edge pressure fluctuation amplitude is smaller when the axial clearance increases. This is also supported by Park et al. ${ }^{7}$ who predicted flow unsteadiness to decrease with increasing axial clearance. Additionally, Chang and Tavoularis ${ }^{15}$ also indicated decreasing unsteadiness in the pressure field before the rotor when the axial clearance was increased. Also Ummiti et al. ${ }^{23}$ found a decrease of pressure fluctuation coefficients in the rotor blade surfaces when the axial clearance increased.

A mechanism which also influences the flow field in the axial clearance is unsteady work, which is defined as the temporal derivative of static pressure. Rose et al. ${ }^{17}$ showed that the unsteady pressure field of the rotor extends upstream and therefore changes the enthalpy of the fluid at the rotor inlet. In another study by Rose et al. ${ }^{18}$, the interaction between the stator wake segments and the rotor suction surface was found to be a source of positive unsteady work. They also proposed that stronger unsteady interaction should be tried to design more efficient machines since the unsteady interaction process has partly the ability to suppress the downstream mixing loss.

In cooled turbine stages the interaction between the coolant and main flow can also affect the efficiency curve shape. Schum et al. ${ }^{24}$ found increasing stage efficiency when the axial clearance increased. They found this to be due to better mixing of coolant and primary flows before the rotor. 
The influence of axial clearance can also depend on the operating conditions. Yamada et al. ${ }^{12}$ studied the effect of axial clearance variation at design and off-design conditions. They found that the efficiency decrement was relatively linear at the off-design, but this behaviour was not reproduced at the design conditions. They concluded that the improved off-design performance was due to stator wake-rotor passage vortex interaction. Also the study of Grönman and Turunen-Saaresti ${ }^{6}$ predicted slightly different efficiency behaviours at design and off-design conditions. The efficiency drop was more drastic at the off-design than it was at the design conditions.

Sharma et al. ${ }^{25}$ studied a 1.5 -stage axial turbine with changing rotor-second stator axial clearance. They found increased midspan loading at the second stator when the axial clearance increased. The midspan loss of the second stator was also decreased when the axial clearance increased.

Sadovnichiy et al..$^{26}$ studied impulse turbines with two different stator vanes. They discussed that the increasing axial clearance has a negative influence on the stage efficiency due to secondary losses. In the turbines they studied, the efficiency did not always decrease with the increasing axial clearance. They stated that with the increased axial clearance, the mixing of the stator wake and the main flow creates losses, but it reduces the wake induced unsteady flow in the rotor and losses related to it. This was found to be the reason for almost constant stage efficiency. For the decreasing stage efficiency, additional losses were also created by the reduced blade loading at the tip region and increased incidence losses at the hub when the axial clearance increased.

Ummiti et al. ${ }^{23}$ studied numerically the effect of axial clearance on the losses in a 1.5-stage axial turbine. Their study predicted that the optimal axial spacing would be $25 \%$ of the stator and rotor axial chord. The stator losses were increasing with increasing axial clearance, but the rotor losses started to increase after $25 \%$ 
axial clearance. Both the rotor and the second stator incidence were found to decrease when the axial clearance was increased mainly because of the decrease in the wake defect.

Kikuchi et al. ${ }^{27}$ studied the performance of axial turbine stage with changing stator-rotor axial clearance. A slight efficiency drop was found with respect to increasing axial clearance. Their measurements indicated increased boundary layer growth in the casing before the rotor when the axial clearance was increased. Similar data close to the hub wall was not available. Also the wake was noticed to diffuse in the circumferential direction with the increasing clearance. It was found that the increasing axial clearance increased entropy at the hub side downstream from the rotor. The extent of fluctuations of rotor passage vortex and secondary flow field near the hub were wider when the axial clearance was increased, while near the casing the effects of unsteadiness were hardly seen with the large clearance.

Based on the current studies, it can be concluded that there are five main loss sources that are affected by the axial clearance: 1 . Stator wake mixing with the main flow, 2. Unsteady interaction between the stator wake and rotor blade, 3. Changing rotor incidence, 4. End wall boundary layer losses, and 5. Stator trailing edge shock related losses.

Despite the numerous axial clearance studies in the literature, it is not currently known what is causing the different efficiency trends for different turbines. It can be expected that the behaviour differences are related to the design parameters of the turbine. This study has two main objectives: the first, to find how the general efficiency trends can help a turbine designer and second, to find design parameters which can be connected to different efficiency trends as a function of stator-rotor axial clearance.

In a previous study of Turunen-Saaresti and Jaatinen, ${ }^{28}$ a linear dependence of several design parameters was studied with the centrifugal compressor tip clearance losses. In this study, a similar approach is chosen to find the possibly correlating design parameters. First, the available data for the turbine efficiency is analysed 
as a function of axial clearance and general recommendations are presented for the designer. Second, the correlations of design parameters are studied to find their importance to the axial clearance related losses. Finally, the correlating parameters are analysed in order to find the physical mechanisms behind the different efficiency curves.

\section{II.Effect of Axial Clearance on the Efficiency Curve Shape}

A typical axial clearance in axial flow turbines is usually $30-40$ per cent of the stator axial chord as was stated by Mokulys et al. ${ }^{5}$ In Figure 1, the efficiency change as a function of axial clearance is given relative to the zero axial clearance. The data has been linearly transformed from the zero clearance, and the linear fits for most negative and positive slopes are given as solid lines. The transformation was necessary to make the data from all references comparable. In general, the trend seems to be that the increase of axial clearance decreases the efficiency. These results clearly illustrate that the efficiency trends are changing between the different designs. In the available literature, many of the studies $^{8,12,15,16}$ predict the optimal axial clearance to be between 0.35 and 0.42 , which can also be considered as a good choice to begin the design process with, reflecting well the design practices. However, it does not necessarily lead to the best available efficiency. 


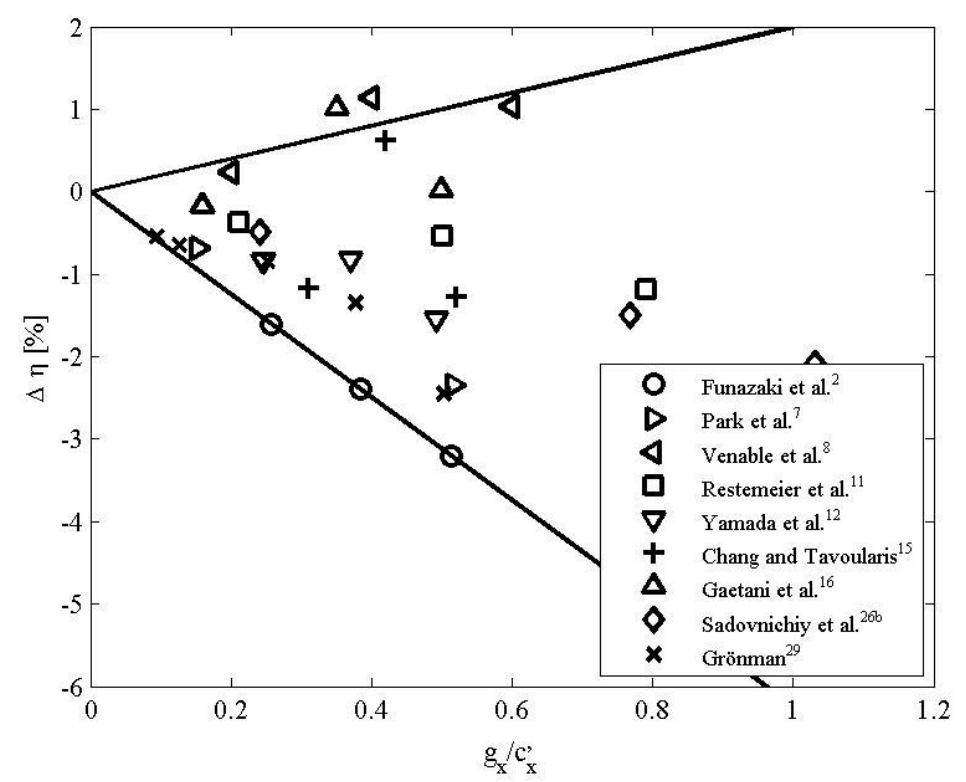

Fig. 1 Efficiency change as a function of axial clearance $g_{x} / c_{x}$,

Some studies have indicated that an optimal axial clearance can be found relative to the stator geometric throat. This is illustrated in Figure 2, in which the efficiency change is given as a function of axial clearance which is non-dimensionalized with the stator geometric throat. Also in this figure, the data has been linearly transformed from the zero clearance in order to make the different turbines comparable. Figure 2 suggests that choosing the axial clearance of $g_{\downarrow} / o^{\prime}=0.9$ is a good choice for initial guess to begin the design process with. This is also close to the optimum value of $g_{x} / o^{\prime}=5 / 6$ found by Gaetani et al..$^{16}$ Also Sadovnichiy et al. ${ }^{26}$ reported values between $0.9-1.3$ to be representative of steam turbine designs in the former Soviet Union. Based on Figures 1 and 2, it can be concluded that some general guidelines can be given for the initial axial clearance. However, for a designer it would be beneficial to have information about the dependency between the curve shape and the typical design parameters. This dependency is studied in the following section. 


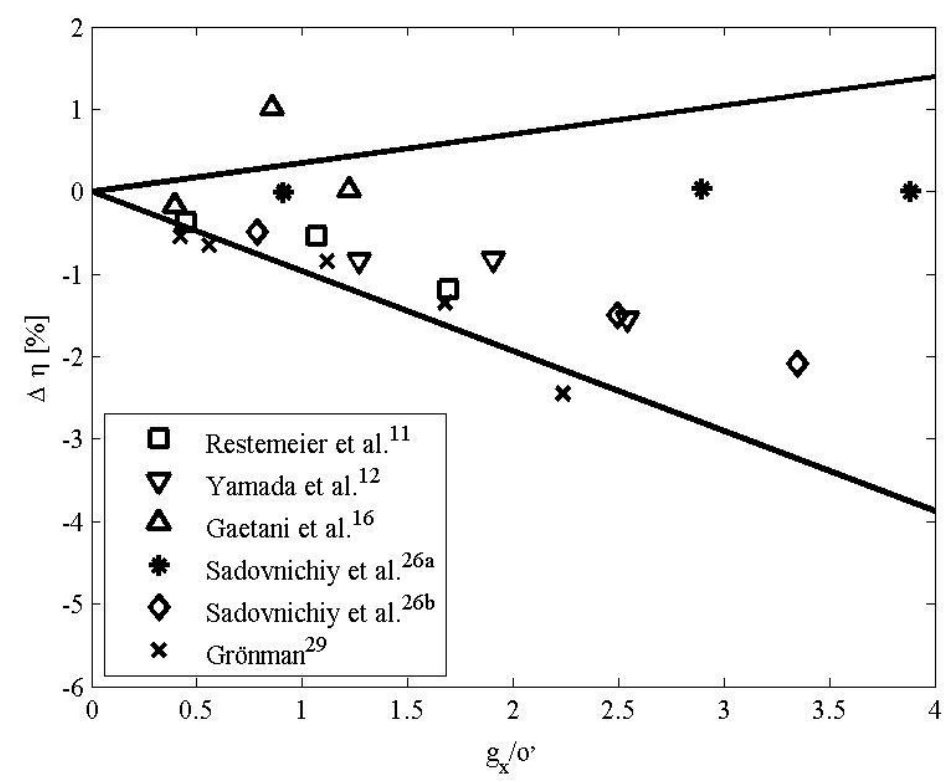

Fig. 2 Efficiency change as a function of axial clearance $g_{x} / o$ '

\section{III.Correlation between Efficiency Slope and Design Parameters}

Several turbine geometries varying in their design values were analysed in order to study the effects of different design parameters. These geometries are described in Table 1. Most of the data is taken from the referred publications or is calculated from the published data, but few parameters are also unpublished data from the authors. The data is partly numerical and partly experimental. The possible uncertainty of the numerical works is also taken into account when evaluating the correlations by analysing experimental data separately. 
Table 1 Studied turbine geometries

\begin{tabular}{|c|c|c|c|c|c|c|c|c|c|c|c|}
\hline Reference & $s^{\prime} / c^{\prime}$ & $s^{\prime \prime} / c^{\prime \prime}$ & $h^{\prime} / c^{\prime}$ & $h^{\prime \prime} / c^{\prime \prime}$ & $h^{\prime} / c_{x}^{\prime}$ & $h^{\prime \prime} / c_{x}^{\prime \prime}$ & $s^{\prime} / c_{x}^{\prime}$ & $s^{\prime \prime} / c_{x}^{\prime \prime}$ & $r_{h}{ }^{\prime \prime} / r_{t}{ }^{\prime}$ & $u / a$ & $b / h^{\prime}$ \\
\hline Funazaki et al. $^{2}$ & - & - & - & - & - & - & - & - & 0.76 & - & - \\
\hline Park et al. ${ }^{7}$ & - & - & 1.01 & 0.95 & - & - & - & - & 0.80 & 0.10 & - \\
\hline Venable et al. ${ }^{8}$ & 0.76 & 0.72 & 0.72 & 1.10 & - & - & - & - & 0.81 & 0.68 & - \\
\hline Restemeier et al. ${ }^{11}$ & 0.90 & 0.98 & 0.95 & 1.40 & 1.45 & 1.77 & 1.37 & 1.24 & 0.82 & 0.30 & 0.005 \\
\hline Yamada et al. ${ }^{12}$ & 0.61 & 0.71 & 1.30 & 2.06 & 1.83 & 2.34 & 0.86 & 0.81 & 0.76 & 0.16 & 0.009 \\
\hline Chang and Tavoularis ${ }^{15}$ & 1.88 & 0.86 & - & - & 0.70 & 1.00 & - & - & 0.82 & - & - \\
\hline Gaetani et al. ${ }^{16}$ & 0.83 & 0.80 & 0.83 & 0.91 & 1.63 & 1.05 & 1.64 & 0.93 & 0.75 & 0.40 & 0.015 \\
\hline Sadovnichiy et al. ${ }^{26 a}$ & 0.72 & 0.51 & 1.86 & 2.75 & - & - & - & - & 0.72 & - & 0.013 \\
\hline Sadovnichiy et al. ${ }^{26 \mathrm{~b}}$ & 0.72 & 0.51 & 1.73 & 2.75 & 2.87 & 3.13 & 1.20 & 0.58 & 0.73 & - & 0.013 \\
\hline Grönman $^{29}$ & 0.70 & 0.68 & 0.40 & 0.67 & 0.61 & 0.72 & 1.08 & 0.72 & 0.84 & 0.87 & 0.022 \\
\hline
\end{tabular}

The first step to find the design parameters which correlate with the efficiency curves was made by assuming linear correlation in all studied cases. The correlation of each fit was estimated by the coefficient of determination $R^{2}$. This approach was chosen, since most of the studied data was suggesting relatively linear efficiency change as shown in Table 2 . The values in Table 2 have been quantified by fitting a linear curve to each data set separately. Therefore, a negative value of the slope of efficiency curve $k$ denotes a turbine which has decreasing efficiency when the axial clearance is increased. 
Table 2 Coefficient of determination for the slope of efficiency based on Fig. 1

\begin{tabular}{lcc}
\hline \hline Reference & $k$ & $R^{2}$ \\
\hline Funazaki et al. $^{2}$ & -6.24 & 1.00 \\
Park et al. $^{7}$ & -4.55 & 1.00 \\
Venable et al. $^{8}$ & 2.00 & 0.66 \\
Restemeier et al. $^{11}$ & -1.39 & 0.89 \\
Yamada et al. $^{12}$ & -2.89 & 0.74 \\
Chang and Tavoularis $^{15}$ & 0.35 & 0.01 \\
Gaetani et al. $^{16}$ & 0.84 & 0.05 \\
Sadovnichiy et al. $^{26 \mathrm{~b}}$ & -2.01 & 1.00 \\
Grönman $^{29}$ & -4.31 & 0.90 \\
\hline \hline
\end{tabular}

Different sensitivity analyses were performed to find the parameters which correlate with the efficiency curve, and the results of the analysis are shown in Table 3. The values of the slope of efficiency from Table 2 were used to represent the shapes of the efficiency curves of different turbines. After this, the correlation between each design parameter from Table 1 and the slope of efficiency was evaluated. A total of eleven parameters were studied. The first row shows the results for the whole data set, and the second for the data with turning velocity Mach numbers $u / a \leq 0.68$. The last row shows the results of the analysis for the measured data.

The analysis of the whole data indicates that with the available data the best linear correlation is found with the stator pitch to chord ratio $\mathrm{s}^{\prime} / \mathrm{c}_{\mathrm{x}}$. This design parameter has the strongest correlation in all analyses, which is a strong indication of its importance. The trend line and the data are shown in Figure 3, which 
suggests that the decreasing stator pitch to chord ratio will lead to more drastic drop in the efficiency as the axial clearance is increased.

When the data is further processed, a relatively good correlation can be found between the slope of efficiency and the turning velocity Mach number with $u / a \leq 0.68$ as illustrated in Figure 4 , indicating that increasing $u / a$ will lead towards turbines that can have improved efficiency as the axial clearance is increased. Additionally, a weaker correlation can be found of the rotor aspect ratio $h$ "'/ $\mathrm{c}_{\mathrm{x}}$ "' when only the measured data is considered, which is also illustrated in Figure 5. In Figure 5, all data is presented, but the trend line is drawn for the measured data only. This design parameter has a different influence on the curve shape compared to the two others presented in Figures 3 and 4 as the increase in the aspect ratio will lead to a steeper drop in the efficiency with increasing axial clearance. One important observation in the analysis is that the experimental data supports the found correlations, and therefore, eliminates the influence of a possible numerical error. These findings are also generally supported by the conclusions which Chang and Tavoularis ${ }^{15}$ made based on their literature review. They concluded that high pressure turbine performance with changing axial clearance depends on the turbine geometry and rotational speed. However, they did not discuss how the above mentioned reasons affect the turbine performance.

Table 3 Coefficient of determination based on regression analysis from Table 2

\begin{tabular}{cccccccccccc}
\hline \hline & $s^{\prime} / c^{\prime}$ & $s^{\prime \prime} / c^{\prime \prime}$ & $h^{\prime} / c^{\prime}$ & $h^{\prime \prime} / c^{\prime \prime}$ & $h^{\prime} / c_{x}{ }^{\prime}$ & $h^{\prime \prime} / c_{x}{ }^{\prime \prime}$ & $s^{\prime} / c_{x}{ }^{\prime}$ & $s^{\prime \prime} / c_{x}{ }^{\prime \prime}$ & $r_{h}{ }^{\prime \prime} / r_{t}{ }^{\prime \prime}$ & $u / a$ & $b / h^{\prime}$ \\
\hline$R^{2}$ & 0.14 & 0.09 & 0.01 & 0.00 & 0.00 & 0.03 & 0.74 & 0.18 & 0.01 & 0.05 & 0.11 \\
$R^{2} u / a \leq 0.68$ & 0.20 & 0.03 & 0.26 & 0.08 & 0.13 & 0.63 & 0.90 & 0.09 & 0.03 & 0.91 & 0.25 \\
$R^{2}$ meas. & 0.45 & 0.12 & 0.46 & 0.60 & 0.13 & 0.63 & 0.90 & 0.09 & 0.00 & 0.95 & 0.25 \\
\hline \hline
\end{tabular}




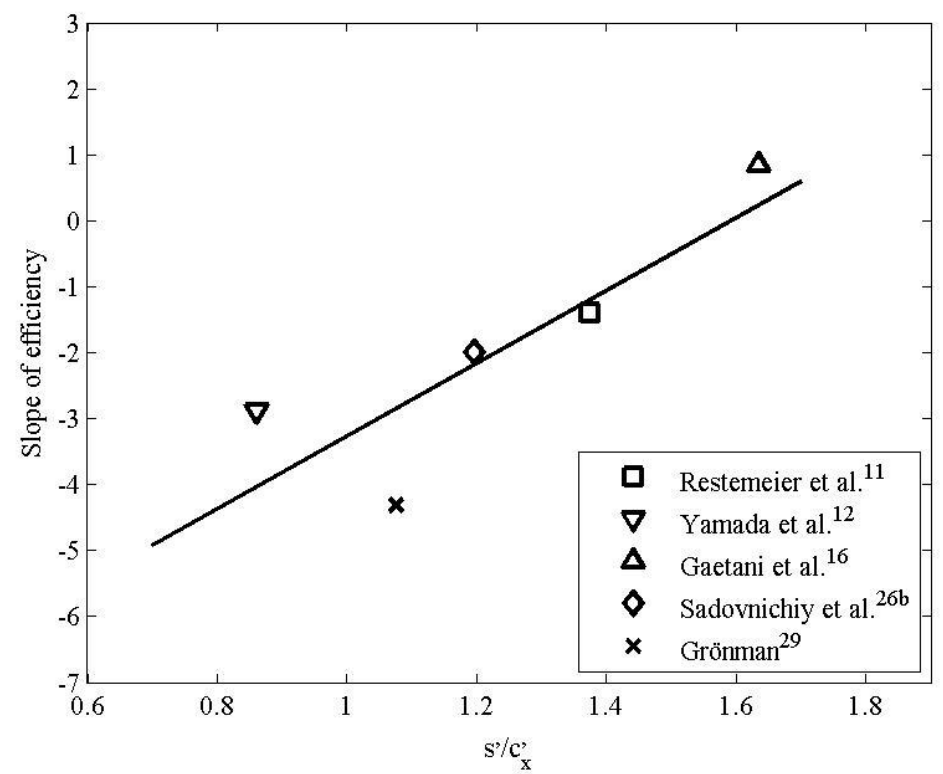

Fig. 3 Correlation between $s^{\prime} / c_{x}$ ' and the slope of efficiency 


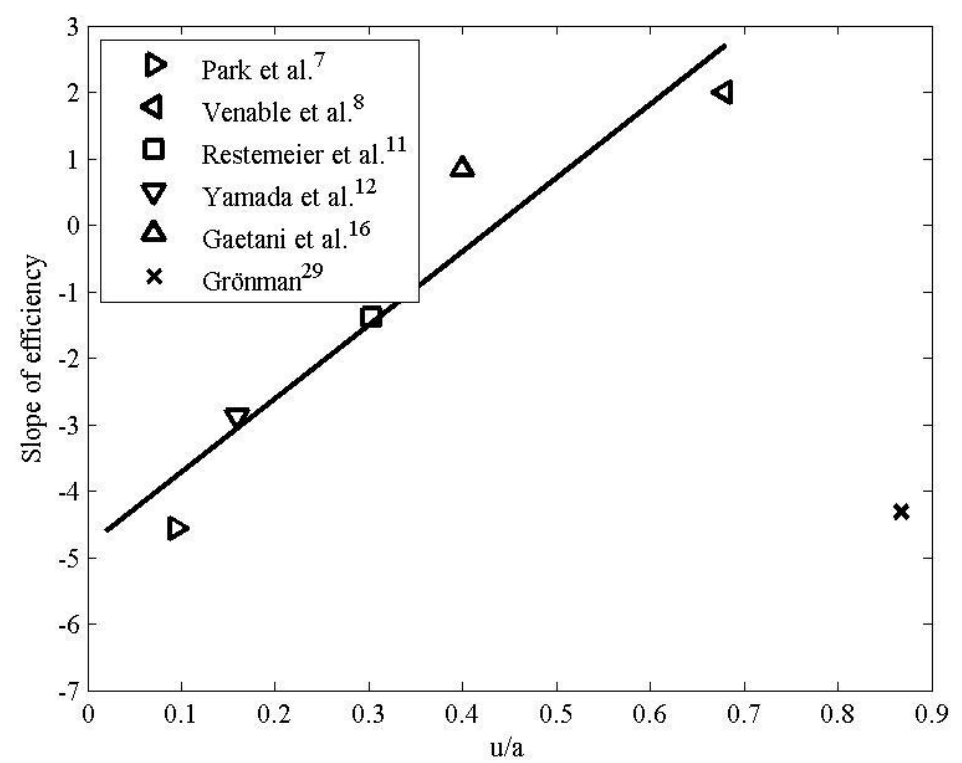

Fig. 4 Correlation between $u / a$ and the slope of efficiency 


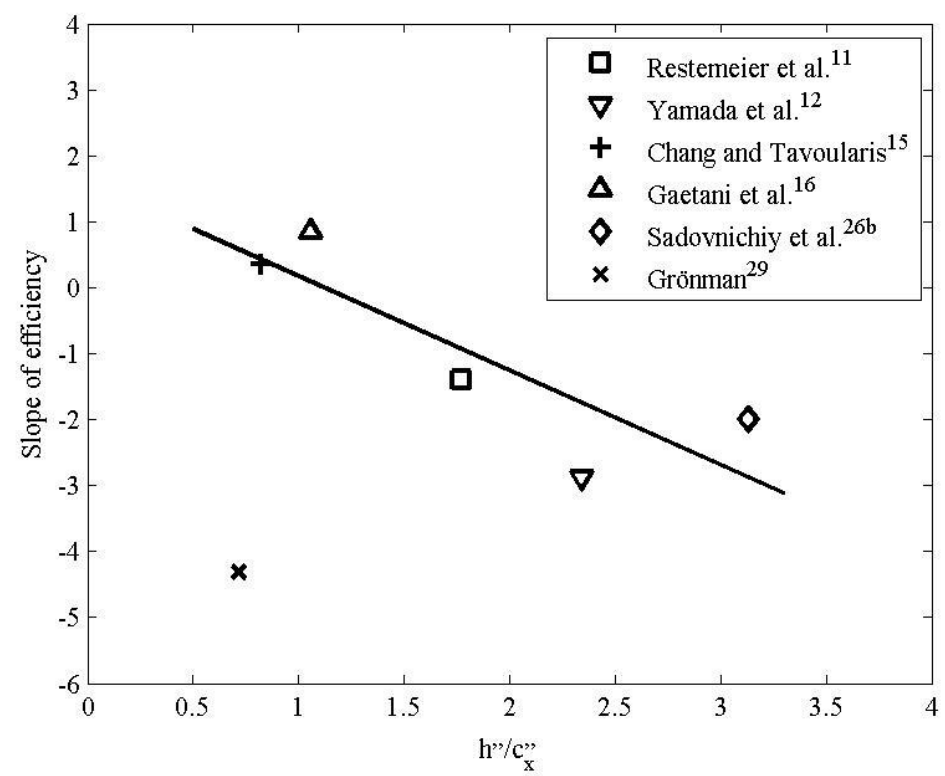

Fig. 5 Correlation between $h$ ''/c $c_{x}$ ' and the slope of efficiency

In Table 4, the chosen design parameters are summarized and evaluated based on the loss mechanisms which are affected by the parameter. First, the loss contribution of the axial clearance is evaluated. The axial clearance contributes to the mixing losses between the blade rows. Larger clearance allows the wake mixing to cause more losses, and from that point of view, a smaller clearance is always better than a larger one. With very large clearances $\left(g_{x} / c_{x}{ }^{\prime} \geq 0.5\right)$ it can be approximated that the mixing process does not produce additional total pressure losses. This approximation can be drawn based on the approximation which was presented by Benner et al. ${ }^{30}$ In their paper, a linear loss development was assumed for the mixing losses as a function of distance from the trailing edge. This does not take the interaction (such as unsteady work ${ }^{17,18}$ ) from the rotor into account, but it can be considered as a fair assumption. 
If the turbine stage is designed at a certain rotor incidence, the changes in the axial clearance size will also lead to changing rotor incidence. ${ }^{13}$ The change of incidence is, however, dependent on the flow path radius change in the axial clearance. With constant radius the change of incidence is mostly located close to the end wall boundary layers. The changes in the incidence can lead to increased rotor profile losses as presented by Benner et al. ${ }^{31}$ Further, the changing incidence will have an effect on tip-clearance losses. ${ }^{32}$ The general influence of the axial clearance to the mean line incidence will, however, be relatively small and neglecting it will not necessarily produce a meaningful error to the one dimensional loss estimation in practical design cases.

The second parameter $s^{\prime} / c_{x}^{\prime}$ contributes to the two main loss sources: wake mixing and wake-rotor interaction. Figure 3 shows that the increasing rotor pitch to axial chord ratio leads towards a positive curve shape. In most of the turbines, in which the stator loading is sufficiently high, the increase of pitch to chord ratio will lead to over proportionally thickened boundary layers and also thicker wakes. Therefore, both stronger wake-rotor interaction and wake mixing will take place. The positive curve shape can then be explained by the stronger wake mixing with increasing $s^{\prime} / c_{x}$ '. These phenomena can be also connected to the findings of Sadovnichiy et al., ${ }^{26}$ according to whom, with the increased axial clearance, the mixing of the stator wake and the main flow creates losses, but it reduces the wake induced unsteady flow in the rotor and the losses related to it. This was found to be the reason for almost constant stage efficiency with a changing clearance.

Turning velocity Mach number can contribute indirectly to the unsteady stator-rotor interaction and, also at the off-design, to the incidence losses. The increasing rotational speed can lead to increased interaction between the stator wake and the rotor blades. With larger clearances the losses related to the unsteady interaction can decrease and therefore lead to improved turbine performance. ${ }^{26}$ However, one should also be 
aware of the possible positive influence of stronger stator-rotor interaction on the downstream mixing loss. ${ }^{18}$ The off-design incidence angles also cause detrimental effects on the performance. To include the unsteady stator-rotor interaction directly into the analysis, for example Strouhal number would be a good parameter.

The importance of hub and shroud side interaction and boundary layer growth in the end walls, found in many studies, ${ }^{2,12,15}$ can be connected to the rotor aspect ratio. Figure 5 indicates that with a constant rotor axial chord, the increase of the blade height would lead into a more drastic drop in the efficiency as the axial clearance is increased. The influence which the aspect ratio has on the stator-rotor interaction is not clear based on the literature, but the increasing boundary layer causes more losses with larger axial clearances. The reason for this could be that the usually negative tip region interaction overcomes the positive hub side interaction with increasing aspect ratios. However, to be certain, more research is needed on this topic.

When extending the analysis to multi-stage turbines, the importance of the stator aspect ratio might also increase. This can, at least partly, be explained by the presence of rotor wakes and the end wall boundary layer development after the rotor. With the presented analysis a relatively weak correlation $\left(R^{2}=0.46\right)$ can also be found with $h^{\prime} / c^{\prime}$ when only the measured data is considered, indicating the potential influence of stator aspect ratio on the axial clearance losses.

Table 4 Design parameter loss contribution

\begin{tabular}{ll}
\hline \hline Parameter & \multicolumn{1}{c}{ Loss contribution } \\
\hline$g_{y} / c_{x}{ }^{\prime}$ & Wake mixing and incidence losses \\
$s^{\prime} / c_{x}$, & Stator wake mixing and wake-rotor interaction losses \\
$u / a$ & Wake-rotor interaction and incidence losses \\
$h^{\prime \prime} / c_{x}$, & End wall boundary layer and wake-rotor interaction losses \\
\hline \hline
\end{tabular}




\section{IV.Conclusions}

Despite the numerous stator-rotor axial clearance studies in the literature, there is a clear lack in the knowledge of the reasons which cause the different trends of efficiency change when altering the axial clearance. The literature review revealed that there are five main loss sources which are affected by the statorrotor axial clearance:

1. Stator wake mixing with the main flow.

2. Unsteady interaction between the stator wake and rotor blade.

3. Changing rotor incidence.

4. End wall boundary layer losses.

5. Stator trailing edge shock related losses.

The available data indicated that the typical axial clearance of 35-42 per cent of the stator axial chord seems to be a safe choice for the designer to begin the design process. However, the optimum value might differ from this, and values lower than 30 per cent could be more optimal in some cases. One existing limiting factor with smaller clearances is the effect on the blade excitation which should be considered in the design process. When the axial clearance is non-dimensionalized with the stator geometric throat, a good starting value for the clearance appears to be $g_{x} / o=0.9$.

The novelty of this study is that, for the first time, the different axial clearance-efficiency curve shapes were linked to certain turbine design parameters and loss mechanisms. The conducted analysis with the available data from the literature revealed that there are four design parameters which have a reasonably good linear correlation with the efficiency curve. The found parameters were linked to the loss mechanisms and 
further analysed. The correlating parameters were: stator-rotor axial clearance $g_{x} / c_{x}^{\prime}$, stator pitch to axial chord ratio $s^{\prime} / c_{x}$, turning velocity Mach number $u / a$, and rotor aspect ratio $h " ' / c_{x}{ }^{\prime \prime}$. It was possible to link four loss sources from five to the correlating design parameters, leaving the stator trailing edge shock related losses out of the analysis. However, with the current available data, there were not enough Mach number data to evaluate this possible dependence, which is also a good avenue for further research. It should be noted that this loss source might be small when compared to wake related losses. Additionally, the stator aspect ratio $h^{\prime} / c^{\prime}$ shows potential correlation with the efficiency curve shape, and its influence can be even more pronounced in multi-stage turbines.

The presented correlating parameters can be used to develop the correlation between the axial clearance losses and the design parameters. For this step, more data should be available, including all the required parameters.

\section{Acknowledgments}

The authors would like to thank Dr. Dongil Chang from the University of Ottawa for providing additional information about their work. The authors would also like to thank the Academy of Finland and Lappeenranta University of Technology. Professor Jari Backman is also acknowledged for giving the opportunity to complete this study.

\section{Funding}

This research received no specific grant from any funding agency in the public, commercial, or not-forprofit sectors. 


\section{References}

1. Gaetani P, Persico G, Dossena V and Osnaghi C. Investigation of the flow field in a HP turbine stage for two stator-rotor axial clearances - Part I: Three-dimensional time-averaged flow field. J Turbomachinery 2007; 129: 572-579.

2. Funazaki K, Yamada K, Kikuchi M and Sato H. Experimental studies on aerodynamic performance and unsteady flow behaviors of a single turbine stage with variable rotor-stator axial clearance: Comparisons with time-accurate numerical simulation. In: Proceedings of ASME Turbo Expo, Montreal, Canada, 14-17 May 2007, paper no. GT2007-27670,pp. 703-711. New York: ASME.

3. Jöcker M. Numerical investigation of the aerodynamic vibration excitation of high-pressure turbine rotors. Doctoral Thesis, Royal Institute of Technology, Stockholm, Sweden, 2002.

4. Baagherzadeh Hushmandi $\mathrm{N}$ and Fransson TH. Effects of multiblocking and axial clearance distance on performance of partial admission turbines: A numerical analysis. J Turbomachinery 2011; 133: 031028.

5. Mokulys T, Congiu F, Rose MG and Abhari RS. Unsteady numerical investigation of the effect of wakes with eddy shedding in different axial turbine aerofoils. Proc IMechE Part A: J Power Energy 2009; 223: 1001-1016.

6. Grönman A and Turunen-Saaresti T. Design and off-design performance of a supersonic axial flow turbine with different stator-rotor axial gaps. Proc IMechE Part A: J Power Energy 2011; 225: 497-503.

7. Park J, Choi M and Baek J. Effects of axial clearance on unsteady secondary flow in one-stage axial turbine. Int J Turbo Jet Eng 2003; 20: 315-333.

8. Venable BL, Delaney RA, Busby JA, Davis, RL, Dorney DJ, Dunn MG, Haldeman CW and Abhari RS. Influence of vane-blade spacing on transonic turbine stage aerodynamics: Part I - Time-averaged data and analysis. J Turbomachinery 1999; 121: 663-672. 
9. van de Wall AG, Kadambi JR and Adamczyk JJ. A transport model for the deterministic stresses associated with turbomachinery blade row interactions. J Turbomachinery 2000; 122: 593-603.

10. Cizmas PGA, Hoenninger CR, Chen S and Martin HF. Influence of inter-row clearance value on turbine losses. Int J Rotating Mach 2001; 7: 335-349.

11. Restemeier M, Jeschke P, Guendogdu Y and Gier J. Numerical and experimental analysis of the effect of variable blade row spacing in a subsonic axial turbine. J Turbomachinery 2013; 135: 021031.

12. Yamada K, Funazaki K, Kikuchi M and Sato H. Influences of axial clearance between blade rows on secondary flows and aerodynamic performance in a turbine stage. In: Proceedings of the ASME Turbo Expo, Orlando, Florida, 8-12 June 2009, paper no. GT2009-59855, pp. 1039-1049. New York: ASME.

13. Gaetani P, Persico G, Dossena V and Osnaghi C. Investigation of the flow field in a HP turbine stage for two stator-rotor axial clearances - Part II: Unsteady flow field. J Turbomachinery 2007; 129: 580-590.

14. Yamada K, Funazaki K, Hiroma K, Tsutsumi M, Hirano Y and Matsuo A. Effect of wake passing on unsteady aerodynamic performance in a turbine stage. In: Proceedings of the ASME Turbo Expo, Barcelona, Spain, 8-11 May 2006, paper no. GT2006-90783, pp. 757-767. New York: ASME.

15. Chang D and Tavoularis S. Effect of the axial spacing between vanes and blades on a transonic gas turbine performance and blade loading. Int J Turbo Jet Eng 2013; 30: 15-31.

16. Gaetani P, Persico G and Osnaghi C. Effects of axial clearance on the vane-rotor interaction in a low aspect ratio turbine stage. J Prop Power 2010; 26: 325-334.

17. Rose, MG., Jenny, P., Gier, J. and Abhari, RS. Experimentally observed unsteady work at inlet to and exit from an axial flow turbine rotor. J Turbomachinery 2013; 135: 061017.

18. Rose, M., Schüpbach, P. and Mansour, M. The thermodynamics of wake blade interaction in axial flow turbines: Combined experimental and computational study. J Turbomachinery 2013; 135: 031015. 
19. Busby JA, Davis RL, Dorney DJ, Dunn MG, Haldeman CW, Abhari RS, Venable BL and Delaney RA. Influence of vane-blade spacing on transonic turbine stage aerodynamics: Part II - Time-resolved data and analysis. J Turbomachinery 1999; 121: 673-682.

20. Jeong E, Park PK, Kang SH and Kim J. Effect of nozzle-rotor clearance on turbine performance. In: Proceedings of the 2006 ASME Joint US-European Fluids Engineering Summer Meeting, Miami, Florida, 17-20 July 2006, paper no. FEDSM2006-98388, pp. 273-278. New York: ASME.

21. Dénos R, Arts T, Paniagua G, Michelassi F and Martelli F. Investigation of the unsteady rotor aerodynamics in a transonic turbine stage. J Turbomachinery 2001; 123: 81-89.

22. Griffin LW and Dorney DJ. Simulations of the unsteady flow through the Fastrac supersonic turbine. $J$ Turbomachinery 2000; 122: 225-233.

23. Ummiti MB, Sitaram N and Prasad BVSSS. Computational investigation of effect of axial spacing on blade row interaction in a 1.5 stage axial flow turbine. Eng Appl Comput Fluid Mech 2009; 3: 56-70.

24. Schum HJ, Szanca EM and Behning FP. Effect of stator-rotor axial clearance on cold-air performance of a turbine with transpiration-cooled stator blading. NASA TM X-67914, 1971.

25. Sharma OP, Stetson GM, Daniels WA, Greitzer EM, Blair MF and Dring RP. Impact of periodic unsteadiness on performance and heat load in axial flow turbomachines. NASA CR-202319, 1997.

26. Sadovnichiy VN, Binner M and Seume JR. The influence of axial clearances and leaned-twisted guide vanes on the shroud leakage and turbine stage efficiency. In: Proceedings of 8th European Conference on Turbomachinery, Graz, Austria, 23-27 March 2009.

27. Kikuchi M, Funazaki K, Yamada K and Sato H. (2008). Detailed studies on aerodynamic performance and unsteady flow behaviors of a single turbine stage with variable rotor-stator axial clearance. Int J Gas Turbine Propul Power Syst 2008; 2: $\quad 30-37$. 
28. Turunen-Saaresti $\mathrm{T}$ and Jaatinen A. Influence of the different design parameters to the centrifugal compressor tip clearance loss. J Turbomachinery 2013; 135: 011017.

29. Grönman, A. Numerical modelling of small supersonic axial flow turbines. Dissertation, Lappeenranta University of Technology, Finland, 2010.

30. Benner MW, Sjolander SA and Moustapha SH. An empirical prediction method for secondary losses in turbines - Part II: A new secondary loss correlation. J Turbomachinery 2006; 128: 281-291.

31. Benner MW, Sjolander SA and Moustapha SH. Influence of leading-edge geometry on profile losses in turbines at off-design incidence: Experimental results and an improved correlation. J Turbomachinery 1997; 119: 193-200.

32. Yamamoto A. Endwall flow/loss mechanisms in a linear turbine cascade with blade tip clearance. $J$ Turbomachinery 1989; 111: 264-275.

\section{Appendix}

\section{Notation}

$a=$ speed of sound at the turbine inlet $[\mathrm{m} / \mathrm{s}]$

$b=$ tip-clearance $[\mathrm{m}]$

$c=$ chord $[\mathrm{m}]$

$c_{x}=$ axial chord $[\mathrm{m}]$

$g_{x}=$ stator-rotor axial clearance $[\mathrm{m}]$

$h=$ height $[\mathrm{m}]$

$k \quad=\quad$ curve slope $[-]$

$N=$ rotational speed $[\mathrm{rpm}]$ 


$$
\begin{aligned}
o & =\text { physical throat width }[\mathrm{m}] \\
r & =\text { radius }[\mathrm{m}] \\
R^{2} & =\text { coefficient of determination }[-] \\
s & =\text { pitch }[\mathrm{m}] \\
u & =\text { maximum blade tip speed }[\mathrm{m} / \mathrm{s}] \\
\Delta \eta & =\text { change of efficiency }[\%]
\end{aligned}
$$

\section{Subscripts}

$$
\begin{aligned}
& \mathrm{h}=\mathrm{hub} \\
& \mathrm{t}=\mathrm{tip}
\end{aligned}
$$

\section{Superscripts}

$$
\begin{aligned}
& \text { ' } \quad \text { stator } \\
& ، \quad=\text { rotor }
\end{aligned}
$$

